\title{
Lévy flights and Lévy-Schrödinger semigroups
}

\author{
Piotr Garbaczewski* \\ Institute of Physics, University of Opole, 45-052 Opole, Poland
}

October 25, 2018

\begin{abstract}
We analyze two different confining mechanisms for Lévy flights in the presence of external potentials. One of them is due to a conservative force in the corresponding Langevin equation. Another is implemented by Lévy-Schrödinger semigroups which induce so-called topological Lévy processes (Lévy flights with locally modified jump rates in the master equation). Given a stationary probability function (pdf) associated with the Langevin-based fractional Fokker-Planck equation, we demonstrate that generically there exists a topological Lévy process with the very same invariant pdf and in the reverse.
\end{abstract}

PACS numbers: 05.40.Jc, 02.50.Ey, 05.20.-y, 05.10.Gg

\section{Motivation}

Lévy flights stand for a nickname of a class of symmetric stable stochastic processes. These non-Gaussian jump-type processes are not yet deeply accommodated within the general statistical physics conceptual and technical imagery. One obstacle is rooted in purely technical reasons - a shortage of explicit analytic solutions, e.g. probability density functions and transition probability densities. Other obstacles may be attributed to somewhat exotic features of Lévy flights, like an existence of arbitrarily small jumps or that of fat tails of the probability density which preclude the existence of moments (in the least of the second one).

There is also a physically motivated obstacle: no physical thermalization mechanisms have ever been proposed for Lévy flights. Moreover, their physical "reason" (origin of noise) appears to be exterior to the physical system, with no reliable kinetic theory background, and therefore no fluctuation-dissipation response theory could have been been set for any stable noise.

To the contrary, the noise "reason" is definitely an intrinsic feature of the environmentparticle coupling in case of the standard Brownian motion, based on the kinetic theory

\footnotetext{
*E-mail: pgar@uni.opole.pl
} 
derivations. All traditional fluctuation-dissipation relationships find their place in the Brownian framework. None of them has been reproduced in the context of Lévy flights.

Nonetheless, Lévy flights remain an active research area, with a definite tendency to expand their field of applicability. A fraction of papers, relevant to our problem (taming Lévy flights via suitable confining potentials) is reproduced in the reference list, [1]-13].

In the present paper we address an apparent discord between two families of jumptype processes which can be be associated with the same type of the free (Lévy noise. Namely, most of current research is devoted to Langevin equation-based derivations, where a deterministic force is perturbed by Lévy (white) noise of interest, [3]-[13]. However, in a number of publications, another class of jump-type processes was introduced under the name of topologically induced super-diffusions, [14]-[18]. As we indicate in below they belong to a broader class of processes governed by Lévy-Schrödinger semigroups. In a different context, they were introduced and investigated in some detail in Refs. [5, 6], c.f. also [2].

Fractional Fokker-Planck equations, inferred respectively for Langevin and topological processes, appear not to describe the same dynamical pattern of behavior. The statement of [16] was that topologically induced fractional Fokker-Planck equation does not portray a situation equivalent to any of standard (e.g. Langevin-based) fractional Fokker-Planck equations and thus does not correspond to any Langevin equation.

Confined Lévy flights have received some attention in the recent literature [8]-[13]. On the other hand, in the discussion of topological Lévy processes main emphasis has been put on their super-diffusive behavior with some neglect of confining effects and the resultant emergence of non-Gibbsian stationary probability densities, [14]-[18].

We address the latter issue and set general confinement criterions for analytically tractable of Cauchy noise-driven processes. To this end, some ideas have been adopted from the general theory of diffusion-type stochastic processes where an asymptotic approach towards equilibrium (stationary density) is one of major topics of interest, [22].

To handle topological Lévy processes we invoke a theoretical framework of the Schrödinger boundary data problem (whose validity is well established in the Brownian context, [19, 21]). We shall demonstrate that topologically induced processes of [14][18] form a subclass of its solutions with a properly tailored dynamical semigroup and its (Feynman-Kac) potential, [5, 6]. That allows to take advantage of the existing mathematical theory of Lévy processes and Lévy-Schrödinger semigroups, [3, 4] and [5, 6, 7], where free Lévy noise generators are additively perturbed by suitable confining potentials. The theory works well for both Gaussian and non-Gaussian processes. (For the record we indicate that, in the Brownian case, the Schrödinger problem incorporates 
the well known transformation of a Fokker-Planck equation into a generalized diffusion equation, [23].)

In Section 2 we give a necessary background for the forthcoming analysis of random motions in terms of dynamical (Schrödinger and next Lévy-Schrödinger) semigroups.

In Section 3 we extend the Schrödinger boundary data problem Section 2 to Lévy flights. In Section 4 we address in detail a number of model cases related to the Cauchy flights, with emphasis on their confinement mechanisms.

\section{Brownian inspiration: Schrödinger semigroups}

\subsection{Gibbsian asymptotics of Smoluchowski processes}

We begin from a brief resume of standard Smoluchowski processes and their generalizations. Since in the present discussion we assume forward drifts to be of the gradient form, our considerations will be limited (albeit with no substantial loss of generality) to one space dimension.

Let us consider $\dot{x}=b(x, t)+A(t)$ with $\langle A(s)\rangle=0,\left\langle A(s) A\left(s^{\prime}\right)\right\rangle=\sqrt{2 D} \delta\left(s-s^{\prime}\right)$. Here, $b(x, t)$ is a forward drift of the process, admitted to be time-dependent, unless we ultimately pass to Smoluchowski diffusion processes where $b(x, t) \equiv b(x)$ for all times and $x \in R$.

Given an initial probability density $\rho_{0}(x)$. We know that the diffusion process drives this density in accordance with the Fokker-Planck equation $\partial_{t} \rho=D \triangle \rho-\nabla(b \cdot \rho)$. We introduce an osmotic velocity field $u=D \nabla \ln \rho$, together with the current velocity field $v=b-u$. The latter obeys the continuity equation $\partial_{t} \rho=-\nabla j$, where $j=v \cdot \rho$ has a standard interpretation of a probability current.

Presently we pass to time-independent drifts of the diffusion process, that are induced by external (conservative, Newtonian) force fields $f=-\nabla V$. One arrives at Smoluchowski diffusion processes by setting

$$
b=\frac{f}{m \beta}=-\frac{1}{m \beta} \nabla V .
$$

This expression accounts for the fully-fledged phase-space derivation of the spatial process, in the regime of large $\beta$. It is taken for granted that the fluctuation-dissipation balance gives rise to the standard form $D=k_{B} T / m \beta$ of the diffusion coefficient.

Let us consider a stationary asymptotic regime, where $j \rightarrow j_{*}=0$. We denote an (a priori assumed to exist, [22]), invariant density $\rho_{*}=\rho_{*}(x)$. Since $v_{*}=0$, there holds

$$
b_{*}=u_{*}=D \nabla \ln \rho_{*} .
$$

However $b=f / m \beta$ by its very definition does not not depend functionally on the 
probability density. Thus $b=b_{*}$ and therefore $\rho_{*}(x)=(1 / Z) \exp \left[-V(x) / k_{B} T\right]$. Our outcome has the Gibbs-Boltzmann form.

Denoting $F_{*} \doteq-k_{B} T \ln Z$, where $Z=\int \exp \left(-V / k_{B} T\right) d x$, we have

$$
\rho_{*}(x)=\exp \left(\left[F_{*}-V(x)\right] / k_{B} T\right) \doteq \exp [2 \Phi(x)]
$$

Here, to comply with the notation of [19, 5] and the forthcoming discussion of a topological generalization of the Brownian motion and next Lévy flights [14]-[18], we have introduced a new potential function $\Phi$ such that $\rho_{*}^{1 / 2}=\exp \Phi$ and $b=2 D \nabla \Phi$.

\subsection{Schrödinger semigroups and their integral kernels}

Given Eq. (3), let us re-define a probability density of the Smoluchowski process as follows

$$
\rho(x, t) \doteq \theta_{*}(x, t) \exp [\Phi(x)]
$$

This allows to map a Fokker-Planck equation into an associated generalized heat equation [23]. Indeed, if $\rho(x, t)$ obeys the Fokker-Planck equation whose drift $b(x)$ has the gradient form, the inferred $\theta_{*}(x, t)$ obeys the parabolic equation

$$
\partial_{t} \theta_{*}=D \Delta \theta_{*}-\mathcal{V} \theta_{*},
$$

provided a compatibility condition

$$
\mathcal{V}(x)=\frac{1}{2}\left(\frac{b^{2}}{2 D}+\nabla b\right)
$$

holds true.

We have here arrived at a contractive semigroup dynamics $\theta_{*}(t)=\left[\exp (-t \hat{H}) \theta_{*}\right](0)$, with the dynamical (Schrödinger) semigroup operator $\exp (-t \hat{H})$ whose generator $-\hat{H}$ involves the familiar (here, $1 / 2 m D$ rescaled) Schrödinger Hamiltonian

$$
\hat{H}=-D \Delta+\mathcal{V}
$$

The initial $t=0$ data read $\theta_{*}(x, 0)$.

For $\mathcal{V}=\mathcal{V}(x), x \in R$, typically expected to be continuous and bounded from below, the operator $\hat{H}=-D \triangle+\mathcal{V}$ is essentially self-adjoint on a natural dense subset of $L^{2}$, and the integral kernel $(s<t)$

$$
k(y, s, x, t)=(\exp [-(t-s) \hat{H}])(y, x)
$$

of the dynamical semigroup $\exp (-t \hat{H})$ is strictly positive. Assumptions concerning an admissible potential may be relaxed. The necessary demands are that $\hat{H}$ is self-adjoint 
and bounded from below. Once we have an integral kernel, the semigroup dynamics takes the form $\theta_{*}(x, t)=\int \theta_{*}(y, s) k(y, s, x, t)$.

The key role, in the transformation of the Fokker-Planck operator and equation into Schrödinger-type operator and (parabolic) equation, is played by the potential $\mathcal{V}$. We recall that $-D \Delta$ is a semigroup generator for the free Brownian motion which yields the heat equation $\partial_{t} \rho=D \Delta \rho$.

Brownian noise perturbations of conservative force fields may be encoded on two equivalent ways. One approach directly employs the Langevin equation, c.f our discussion of Smoluchowski processes. It is the deterministic force field $\sim-\nabla V$ which one additively perturbs by white noise.

Another approach is based on the classic Feynman-Kac formula which uniquely assigns a dynamical semigroup kernel (and dynamics) to a concrete potential $\mathcal{V}(x)$ :

$$
k(y, s, x, t)=\int \exp \left[-\int_{s}^{t} \mathcal{V}(X(u), u) d u\right] d \mu[s, y \mid t, x] .
$$

In the above we integrate the $\exp \left[-\int_{s}^{t} \mathcal{V}(X(u), u) d u\right]$ weighting factor with respect to the conditional Wiener measure i.e. along all sample paths (Brownian trajectories) of the Wiener process $X(t)$ which connect $y$ with $x$ in time $t-s ; X(t)$ stands for the random variable of the Wiener process.

\subsection{Schrödinger's boundary data problem}

Schrödinger semigroups naturally appear in the framework of so-called Schrödinger boundary data problem, [19]. We assume to have a priori given two probability densities

$\rho(x, 0)$ and $\rho(x, T), T>0$ and attempt to construct a Markov process that interpolates between these boundary data.

The major outside input is a definite choice of a bounded strictly positive and continuous integral kernel $k(x, s, y, t)$, for all times in the interval (possibly infinite) $0 \leq s<t \leq T$. Our considerations are restricted to Feynman-Kac kernels and so to Schrödinger semigroups. Each semigroup is selected by prescribing the Feynman-Kac potential $\mathcal{V}$, c.f. Eqs. (7) and (9), which needs to be a continuous function. For each concrete choice of the semigroup kernel, cf. [19, 20].

By denoting (notice that we have here combined both forward and backward propagation scenarios in the time interval $[0, T])$ :

$$
\begin{aligned}
\theta_{*}(x, t) & =\int f(z) k(z, 0, x, t) d z, \\
\theta(x, t) & =\int k(x, t, z, T) g(z) d z
\end{aligned}
$$


where functions $f(x)$ and $g(x)$ are suitable inital/terminal data, it follows that for all $0 \leq s<t \leq T$ there holds

$$
\rho(x, t) \doteq \theta(x, t) \theta_{*}(x, t)=\int p(y, s, x, t) \rho(y, s) d y .
$$

Here

$$
p(y, s, x, t)=k(y, s, x, t) \frac{\theta(x, t)}{\theta(y, s)}
$$

is the transition probability density of the pertinent Markov process that interpolates between $\rho(x, 0)$ and $\rho(x, T)$. We note that $\theta_{*}(x, 0)=f(x)$ while $\theta(x, T)=g(x)$.

In case of the free evolution, by setting $\theta(x, t)=\theta \equiv$ const, we effectively transform an integral kernel $k$ of the $L^{1}(R)$ norm-preserving semigroup into a transition probability density $p$ of the Markov stochastic process, e.g. the familiar heat kernel.

Our $\theta$ and $\theta_{*}$ are defined up to constant factors. This freedom is limited by the demand that $\theta \cdot \theta_{*} \doteq \rho$ actually is a probability density of the pertinent Markov process.

We emphasize that in addition to the semigroup dynamics, to secure the uniqueness of an interpolating Markov process, we need an auxiliary motion rule, c.f. [19], (propagating backwards in time the prescribed terminal function $\theta(x, T)$ )

$$
\partial_{t} \theta=-D \Delta \theta+\mathcal{V} \theta
$$

For time-independent $\theta=\theta(x)=\exp \Phi(x)$ we note that $\mathcal{V}=D \Delta \theta / \theta$. Taking into account the definition of the forward drift $b=2 D \nabla \theta / \theta$, [19], we end up with the previous compatibility condition (6).

In case of Smoluchowski processes we encounter asymptotic invariant densities, [22]. Accordingly, $\theta \sim \rho_{*}^{1 / 2}$ and thus

$$
\mathcal{V}=D \frac{\Delta \rho_{*}^{1 / 2}}{\rho_{*}^{1 / 2}} .
$$

This result derives from the semigroup argument alone and does not rely on the Gibbsian form of $\rho_{*}$.

\section{$3 \quad$ Lévy-Schrödinger semigroups}

\subsection{Stable noise and its generator}

The Schrödinger boundary data problem is amenable to an immediate generalization to infinitely divisible probability laws which induce contractive semigroups (and their kernels) for general Gaussian and non-Gaussian noise models, [5] and [6]-[15]. A subclass of stable probability laws contains a subset that is associated in the literature with the concept of Lévy flights. 
Instead of the semigroup generators proper, we shall employ the rescaled Hamiltonians (true generators have an opposite sign) of the form $\hat{H}=F(\hat{p})$, where $\hat{p}=-i \nabla$ stands for the momentum operator (up to the scaled away $\hbar$ or $2 m D$ factor), and for $-\infty<k<+\infty$, the function $F=F(k)$ is real valued, bounded from below and locally integrable. Then, for a function $f(x)$ in the domain of the semigroup operator, we have

$$
[\exp (-t \hat{H}) f](x)=[\exp (-t F(p)) \tilde{f}(p)]^{\vee}(x)
$$

where the superscript $\vee$ denotes the inverse Fourier transform, and $\tilde{f}$ stands for the Fourier transform of $f$. Let us set

$$
k_{t}=\frac{1}{\sqrt{2 \pi}}\left[\exp (-t F(p)]^{\vee} .\right.
$$

The action of $\exp (-t \hat{H})$ can be given in terms of a convolution (i.e. by means of an integral kernel $\left.k_{t} \equiv k(x-y, t)=k(y, 0, x, t)\right)$ :

$$
\exp (-t \hat{H}) f=[\exp (-t F(p)) \tilde{f}(p)]^{\vee}=f * k_{t}
$$

where

$$
(f * g)(x):=\int_{R} g(x-z) f(z) d z .
$$

We shall restrict considerations only to those $F(p)$ which give rise to positivity preserving semigroups: if $F(p)$ satisfies the celebrated Lévy-Khintchine formula, then $k_{t}$ is a positive measure for all $t \geq 0$. The most general case refers to a combined contribution from three types of processes: deterministic, Gaussian, and the jumptype process.

We recall that a characteristic function of a random variable $X$ completely determines a probability distribution of that variable. If this distribution admits a density we can write $E[\exp (i p X)]=\int_{R} \rho(x) \exp (i p x) d x$ which, for infinitely divisible probability laws, gives rise to:

$$
F(p)=-\int_{-\infty}^{+\infty}\left[\exp (i p y)-1-\frac{i p y}{1+y^{2}}\right] \nu(d y)
$$

where $\nu(d y)$ stands for the appropriate Lévy measure. The corresponding non-Gaussian Markov process is characterized by

$$
E\left[\exp \left(i p X_{t}\right)\right]=\exp [-t F(p)]
$$

Accordingly, the contractive semigroup generator follows (keep the minus sign in memory) from: $F(\hat{p})=\hat{H}$. 
For the sake of clarity we restrict further considerations to non-Gaussian random variables whose probability densities are centered and symmetric, e.g. a subclass of stable distributions characterized by

$$
F(p)=\lambda|p|^{\mu} \Rightarrow \hat{H} \doteq \lambda|\Delta|^{\mu / 2}
$$

Here $\mu<2$ and $\lambda>0$ stands for the intensity parameter of the Lévy process. To account for the interval $0 \leq \mu \leq 2$ boundaries, one should rather employ $(-\Delta)^{\mu / 2}$ instead of $|\Delta|^{\mu / 2}$, since $-\Delta$ is a positive operator.

The fractional Hamiltonian $\hat{H}$, which is a pseudo-differential operator, by construction is positive and self-adjoint on a properly tailored domain. A sufficient and necessary condition for both these properties to hold true is that the probability density of the Lévy process is symmetric, [3].

The associated jump-type dynamics is interpreted in terms of Lévy flights. In particular

$$
F(p)=\lambda|p| \rightarrow \hat{H}=F(\hat{p})=\lambda|\nabla| \doteq \lambda(-\Delta)^{1 / 2}
$$

refers to the Cauchy process, see e.g. [5, 6, 7].

The pseudo-differential Fokker-Planck equation, which corresponds to the fractional Hamiltonian (22) and the fractional semigroup $\exp \left(-t \hat{H}_{\mu}\right)=\exp \left(-\lambda|\Delta|^{\mu / 2}\right)$, reads

$$
\partial_{t} \rho=-\lambda|\Delta|^{\mu / 2} \rho
$$

to be compared with the heat equation $\partial_{t} \rho=+D \Delta \rho$.

\subsection{Response to external potentials: stationary densities}

\subsubsection{Langevin scenario}

In case of jump-type (Lévy) processes a response to external perturbations by conservative force fields appears to be particularly interesting. On the one hand, one encounters a widely accepted reasoning, [8]-[13] where the Langevin equation, with additive deterministic and Lévy "white noise" terms, is found to imply a fractional Fokker-Planck equation, whose form faithfully mimics the Brownian version, e.g. (c.f. [8], see also [7])

$$
\dot{x}=b(x)+A^{\mu}(t) \Longrightarrow \partial_{t} \rho=-\nabla(b \cdot \rho)-\lambda|\Delta|^{\mu / 2} \rho
$$

where we keep the notation $b=f / m \beta, f=-\nabla V$ of Eq. (1).

We emphasize a difference in sign in the second term, if compared with Eq. (4) of Ref. [8]. There, the minus sign is absorbed in the adopted definition of the (Riesz) fractional derivative. Apart from the formal resemblance of operator symbols, we do not directly employ fractional derivatives in our formalism. 
The validity of (24) and temporal details of an approach towards an asymptotic invariant density were investigated for the Cauchy-Ornstein-Uhlenbeck proces [7]. That safely extends to more general stable (symmetric) OU processes, [8] whose asymptotic behavior directly follows from the Fourier transform of $\rho(x, t)$.

In contrast to the standard Gaussian case (folk theorem: all solutions of a given Fokker-Planck equation have a common asymptotic behavior), there are no general mathematically rigorous statements about the asymptotic behavior of solutions of a general fractional Fokker-Planck equation. Specifically, this comment pertains to whether (if at all) and how (detailed convergence estimates) invariant (stationary, equilibrium) densities are asymptotically approached in the course of a nonlinear (symmetric) stable process. Anyway, for monomial drifts, analytic forms of associated invariant densities were explicitly elaborated in the presence of Cauchy noise in Refs. [10, 12, 13].

It is also well known that, given the drift $b$ potential $V(x),(1)$, an invariant density for a confined Lévy flight does not show any connection with the Gibbsian exponential of the form (3). Hence, the usefulness of the transformation (4) comes under scrutiny in the presence of Lévy noise.

\subsubsection{Feynman-Kac (topological) route}

It is a possible to account for external perturbations by means of Lévy-Schrödinger Hamiltonians, [15] and [5, 6] where a potential function $\mathcal{V}(x)$ appears as a necessary ingredient. Assuming that its functional form guarantees that

$$
\hat{H}_{\mu} \doteq \lambda|\Delta|^{\mu / 2}+\mathcal{V}
$$

is self-adjoint and positive in a suitable Hilbert space, we can consistently introduce the Lévy-Schrödinger semigroup $\exp \left(-t \hat{H}_{\mu}\right)$ and the fractional relative of the generalized diffusion equation:

$$
\partial_{t} \theta_{*}=-\lambda|\Delta|^{\mu / 2} \theta_{*}-\mathcal{V} \theta_{*} .
$$

The time-adjoint equation (compare e.g. (15)) has the form

$$
\partial_{t} \theta=\lambda|\Delta|^{\mu / 2} \theta+\mathcal{V} \theta
$$

Clearly, we have here reproduced the general theoretical framework of the Schrödinger boundary data problem, where $\theta^{*}(x, t) \theta(x, t)=\rho(x, t)$ stands for a probability density of an associated Markov process.

Let $\rho_{*}(x)$ be a stationary (invariant, equilibrium) probability density of the pertinent process. With $\theta(x, t) \equiv \theta(x)=\exp [\Phi(x)]$, we can mimic the trial decomposition ansatz, Eq. (4) $\theta_{*}=\rho \exp (-\Phi)$. 
If we set $\exp [\Phi(x)]=\rho_{*}^{1 / 2}(x)$, then Eq. (27) takes the form of the compatibility condition, akin to that of Eq. (14):

$$
\mathcal{V}=-\lambda \frac{|\Delta|^{\mu / 2} \rho_{*}^{1 / 2}}{\rho_{*}^{1 / 2}} .
$$

This identity should be compared with Eq. (8) in Ref. [17], where an analogous effective potential (up to a systematic sign difference) was deduced for the fractional Lévy-Schrödinger type equation, in the study of Lévy flights in inhomogeneous media.

In view of (26) and (27) we have a continuity equation with an explicit fractional input

$$
\partial_{t} \rho=\theta \partial_{t} \theta^{*}=-\lambda(\exp \Phi)|\Delta|^{\mu / 2}[\exp (-\Phi) \rho]-\mathcal{V} \cdot \rho \doteq-\nabla j
$$

Let us make cosmetic changes $\Phi \rightarrow-V / 2 k_{B} T$, c.f. Eqs. (3) and (17) for comparison). Next we take advantage of $\rho=\theta_{*} \theta$ with $\theta=\exp (-\beta V / 2)$ and Eq. (28). By setting $\lambda=1$ and $\beta=1 / k_{B} T$, we give Eq. (35) a familiar form of the transport equation previously introduced in a number of papers:

$$
\partial_{t} \rho=-\exp (-\beta V / 2)|\Delta|^{\mu / 2} \exp (\beta V / 2) \rho+\rho \exp (\beta V / 2)|\Delta|^{\mu / 2} \exp (-\beta V / 2),
$$

c.f. formula (6) in [17], formula (5) in [18] and formula (36) in [14]. There, the investigated process was named a topologically induced super-diffusion. We point out a systematic sign difference between our $|\Delta|^{\mu / 2}$ and the corresponding fractional derivative $\Delta^{\mu / 2}$ of [14, 17, 18]. Graphically these symbols look similar, but have different origin.

The major observation at this point is, that topological Lévy (specifically, Cauchy) processes have been embedded into a well developed mathematical framework of Ref. [6]. Therefore, we can reconcile any specific choice of $\theta \sim \rho_{*}^{1 / 2}$ with minimal requirements upon the properties of $\mathcal{V}$, Eq. (28), that guarantees the existence of LévySchrödinger semigroup and thence of the fractional transport equations (29), (30).

\subsubsection{A discord and its analysis}

The puzzling point, raised in [14], is that for Lévy processes in external force fields, the Langevin approach yields a continuity (e.g. fractional Fokker-Planck) equation in a form

$$
\partial_{t} \rho=-\nabla\left(-\frac{\nabla V}{m \beta} \rho\right)-\lambda|\Delta|^{\mu / 2} \rho
$$

that is very different from the previous fractional transport equation (29).

The conclusion of Refs. [14]-[18] was that, while assuming $\Phi \sim-V$ where $V$ is the external force potential (up to inessential factors), the two transport equations (29) and (31) are plainly incompatible. Eq. (31) seems not to correspond to any standard 
Langevin equation with Lévy noise term and $b=-\nabla V / m \beta$ as its deterministic part and in reverse.

Apart from the verbal statement this puzzling discrepancy has not been explored in more depth. We shall partly fill this gap in below.

Let us take the fractional Fokker-Planck equation (31) for granted. Assume that $\rho(x, t) \rightarrow \rho_{*}(x)$, as $t \rightarrow \infty$. Let the invariant probability density of the fractional

equation (31) determines $\Phi$ through $\exp [\Phi(x)]=\rho_{*}^{1 / 2}(x)$. We know that in case of Lévy noise $\rho_{*}(x)$ does not have a Gibbsian structure $\rho_{*} \sim \exp \left(-V / k_{B} T\right)$, with $V$ being the drift potential.

The general problem to be addressed is:

(i) choose a functional form of $V(x)$ and thus the drift of the Langevin-type process,

(ii) infer an invariant density $\rho_{*}$ that is compatible with the fractional Fokker-Planck equation (31),

(iii) given $\rho_{*}$, deduce the corresponding Feynman-Kac (e.g. dynamical semigroup) potential $\mathcal{V}$ by means of (28): the two dynamical scenarios (29) and (31) would thus share a common stationary density,

(iv) use $\mathcal{V}$ in (28),(29) and verify whether the "topologically induced dynamics" is affine, if at all, to that associated with (31) (and thus to the underlying Langevin equation with Lévy noise),

(v) check an asymptotic behavior of $\rho(x, t)$ in both scenarios (29) and (31) to find possible differences in the speed (convergence time rate) with which the common invariant density $\rho_{*}(x)$ is approached.

(vi) repeat the procedure in reverse by starting from (iii) and then deduce the drift for the Langevin equation with Lévy noise; next compare the dynamical scenarios (29) and (31) for any common initial probability density.

In below, we shall mostly concentrate on the above points (i)-(iii). However, their reverse (vi) will receive some attention as well. We note that problems (iv)-(vi) need more detailed computer-assisted analysis, that is postponed to a future publication.

\section{Processes induced by Cauchy noise: Invariant density vs semigroup (Feynman-Kac) potential}

In view of serious technical difficulties, we shall not attempt a fully fledged solution to the just formulated problem, for any symmetric stable jump-type process and any conceivable drift. Instead, we turn our attention to situations where explicit functional forms of invariant densities are available.

Most of them were inferred in connection with Cauchy noise, [5, 7], 8]-[13]. In particular, attention has been paid to confining properties of various monomial drifts upon 
the Cauchy noise. On the other hand, Lévy flights through a "potential landscape" (topological processes of [14]-[18]) were interpreted as (enhanced) super-diffusions and an issue of possible asymptotic densities has not been significantly developed.

For a pseudo-differential operator $|\Delta|^{\mu / 2}$, the action on a function from its domain is greatly simplified, in view of the properties of the Lévy measure $\nu_{\mu}(d x)$. We have [5, 16, 6, 15, 13]:

$$
\begin{gathered}
\left(|\Delta|^{\mu / 2} f\right)(x)=-\int_{R}\left[f(x+y)-f(x)-\frac{y \nabla f(x)}{1+y^{2}}\right] \nu_{\mu}(d y) \\
\Downarrow \\
\left(|\Delta|^{\mu / 2} f\right)(x)=-\int[f(x+y)-f(x)] \nu_{\mu}(d y) .
\end{gathered}
$$

The Cauchy-Lévy measure, associated with the Cauchy semigroup generator $|\Delta|^{1 / 2} \doteq$ $|\nabla|$, reads

$$
\nu_{1 / 2}(d y)=\frac{1}{\pi} \frac{d y}{y^{2}}
$$

By changing an integration variable $y \rightarrow z=x+y$, we give Eq. (38) the familiar form

$$
(|\nabla| f)(x)=-\frac{1}{\pi} \int \frac{f(z)-f(x)}{|z-x|^{2}} d z
$$

where $1 / \pi|z-x|^{2}$ has an interpretation of an intensity with which jumps of the size $|z-x|$ occur. Once we set $f=\exp \Phi$, the "topologically induced" jump-type process of Refs. [14]-18] is in fact obtained.

\subsection{Ornstein-Uhlenbeck-Cauchy process}

In case of the Ornstein-Uhlenbeck-Cauchy (OUC) process, the drift is given by $b(x)=$ $-\gamma x$, and an asymptotic invariant density associated with

$$
\partial_{t} \rho=-\lambda|\nabla| \rho+\nabla[(\gamma x) \rho]
$$

reads:

$$
\rho_{*}(x)=\frac{\sigma}{\pi\left(\sigma^{2}+x^{2}\right)}
$$

where $\sigma=\lambda / \gamma$, c.f. Eq. (9) in Ref. [7].

Note that a characteristic function of this density reads $-F(p)=-\sigma|p|$ and gives account of a non-thermal fluctuation-dissipation balance. The modified noise intensity parameter $\sigma$ is a ratio of an intensity parameter $\lambda$ of the free Cauchy noise and of the friction coefficient $\gamma$.

For the Cauchy random variable $X_{t}$ we have $E\left[\exp \left(i p X_{t}\right)\right]=\exp (t \lambda|p|)$ where $\lambda$ stands for an intensity of the Cauchy process. The corresponding (time-dependent) probability density has the form (36) with $\sigma \sim t \lambda$, e. g. $\rho(x, t)=\lambda t / \pi\left[(\lambda t)^{2}+x^{2}\right]$. 
Here, $\sigma$ and likewise $t \lambda$ play the role of scale parameters which specify the halfwidth of the Cauchy density at its half-maximum. Since $t \lambda$ grows monotonically, the corresponding free Cauchy noise probability density flattens and its maximum drops down with the flow of time.

In view of $\sigma=\lambda / \gamma$, the frictional drift $-\gamma x$ may stop the "flattening" of the probability distribution and stabilize the density at quite arbitrary shape (with respect to its maximum and half-maximum related half-width), by manipulating $\gamma$. For example, $\gamma \gg 1$ would induce a significant shrinking of the distribution $\rho_{*}$, if compared to the reference (free noise) probability density at any time $t \sim 1 / \lambda$. In parallel, a maximum value of the density would increase: $1 / \pi \lambda \rightarrow \gamma / \pi \lambda$.

Clearly, large friction has a confining effect on Cauchy noise. Confined Lévy flights, and specifically confined Cauchy flights, were analyzed before in [10]-[13] with the aim to produce explicit invariant probability densities in the presence of external (confining) forces. Their properties proved to be quite interesting for monomial and polynomial choices of $V(x)$.

To deduce the Feynman-Kac potential $\mathcal{V}$ for the OUC process, given an invariant density $\rho_{*}$, Eq. (36), we need to evaluate

$$
\mathcal{V}(x)=\frac{\lambda}{\pi}\left(\sigma^{2}+x^{2}\right)^{1 / 2} \int\left[\frac{1}{\sqrt{\sigma^{2}+(x+y)^{2}}}-\frac{1}{\sqrt{\sigma^{2}+x^{2}}}\right] \frac{d y}{y^{2}} .
$$

In the notation $a=\sigma^{2}+x^{2}, b=2 x, R(y)=\sigma^{2}+(x+y)^{2}$ the indefinite integral reads, [24]:

$$
\frac{\lambda}{\pi} \int\left[\frac{\sqrt{a}}{y^{2} \sqrt{R(y)}}-\frac{1}{y^{2}}\right] d y=\frac{\lambda}{\pi}\left[-\frac{\sqrt{R(y)}}{y \sqrt{a}}+\frac{b}{2 a} \operatorname{Arsh}\left(\frac{2 a+b y}{2 \sigma|y|}\right)+\frac{1}{y}\right] .
$$

Because of the singularity at $y=0$, we must handle the integral in terms of its principal value, i.e. by resorting to $\int \rightarrow \int_{-\infty}^{-\epsilon}+\int_{\epsilon}^{+\infty}$, and next performing the $\epsilon \rightarrow 0$ limit.

Taking into account that $\operatorname{Arsh} x \equiv \ln \left(x+\sqrt{1+x^{2}}\right)$, 26], we ultimately get

$$
\mathcal{V}(x)=\frac{\lambda}{\pi}\left[-\frac{2}{\sqrt{a}}+\frac{x}{a} \ln \frac{\sqrt{a}+x}{\sqrt{a}-x}\right] .
$$

$\mathcal{V}(x)$ is bounded both from below and above, with the asymptotics $(2 /|x|) \ln |x|$ at infinities, well fitting to the general mathematical construction of (topological) Cauchy processes in external potentials, [6].

Accordingly, we know for sure that there exists a topological Cauchy process with the Feyman-Kac potential $\mathcal{V}$, Eq. (39), whose invariant density coincides with that for the Langevin-supported OUC process.

At the moment, we have nothing to say about a detailed time-dependent behavior of the topological process and a particular scenario of an approach towards the invariant 
density (equilibrium) in the large time regime. The two considered jump-type processes, whose dynamics is embodied respectively in the fractional Fokker-Planck equation and in the Lévy-Schrödinger semigroup (topological case) dynamics definitely stay in affinity, since they share a common invariant density. In the near-equilibrium regime, any dynamical distinction between these motion scenarios, becomes immaterial. However, their detailed dynamical behavior far-from-equilibrium might be different and this issue deserves further exploration.

There is no faithful Langevin-type representation of a topological process and in reverse, even though an invariant density is common for both. Nonetheless, we have demonstrated that by staring from a common initial probability density, the two (Langevin and dynamical semigroup) motion scenarios may, in principle, end up at a common invariant density.

\subsection{Confined "topological" Cauchy process}

Neither the OUC process nor its topological relative are confined. For the Cauchy density, the second moment is nonexistent. We shall verify the outcome of the OUC discussion for Cauchy-type processes whose invariant densities admit the second moment. Let us consider the quadratic Cauchy density:

$$
\rho_{*}(x)=\frac{2}{\pi} \frac{1}{\left(1+x^{2}\right)^{2}} .
$$

The action of $|\nabla|$ upon this density can be evaluated by recourse to the free Cauchy evolution.

We note that $(1 / \sqrt{2 \pi}) \rho_{*}^{1 / 2}=(1 / \pi) /\left(1+x^{2}\right)$ actually is the Cauchy probability density. Let us consider $f(x)=\rho_{*}^{1 / 2}$ as the initial data for the free Cauchy evolution $\partial_{t} f=\lambda|\nabla| f$. This takes $f(x)$ into

$$
f(x, t)=\frac{2}{\pi} \frac{1+\lambda t}{\left[(1+\lambda t)^{2}+x^{2}\right]} .
$$

Since

$$
\lambda|\nabla| f=-\lim _{t \downarrow 0} \partial_{t} f
$$

we end up with

$$
\mathcal{V}(x)=\frac{\lim _{t \downarrow 0} \partial_{t} f}{f}(x)=\lambda \frac{x^{2}-1}{x^{2}+1} .
$$

The shape of this potential is quite inspiring. A minimum $-\lambda$ is achieved at $x=0$, $\mathcal{V}=0$ occurs for $x= \pm 1$, a maximum $+\lambda$ is reached at $x \rightarrow \pm \infty$.

The potential is bounded both from below and above, hence can trivially be made non-negative (add $\lambda$ ). Therefore, the invariant density (40) is fully compatible with the general construction of the Cauchy-Schrödinger semigroup and the induced jump-type 
process, c.f. Corollary 2, pp. 1071 in [6]. This topological Cauchy process is induced by the Cauchy generator plus a potential function $\mathcal{V}$ given by Eq. (43). c.f. Corollary 2, pp. 1071 in [6]. The process is of the jump-type and can be obtained as an $\epsilon \downarrow 0$ limit of a step process, e.g. jump process whose jump size is bounded from below by $\epsilon>0$ but unbounded from above.

In connection with the problem (vi) o Section 3.2.3 let us note that if the quadratic Cauchy density (40) would actually stand for a stationary density of the fractional Fokker-Planck equation with a drift Eq. (31), then we should have:

$$
\partial_{t} \rho_{*}=0=-\nabla\left(b \rho_{*}\right)-\gamma|\nabla| \rho_{*} .
$$

Therefore the drift function, if any, may be deduced by means of an indefinite integral:

$$
b(x)=-\frac{\gamma}{\rho_{*}(x)} \int\left(|\nabla| \rho_{*}\right)(x) d x .
$$

If we equate 0 , otherwise arbitrary integration constant, we have associated with $\rho_{*}(x)=2 / \pi\left(1+x^{2}\right)^{2}$ an admissible drift function:

$$
b(x)=-\frac{\gamma}{8}\left(x^{3}+3 x\right) .
$$

Thus, if we wish to deal with the Langevin process associated with the quadratic Cauchy density (40), the proper drift form is given above.

\section{3 "Topological" Cauchy family}

We may consider various probability densities as trial ones. Let us pay attention to a broader class of densities that bear close affinity with the Cauchy noise. With a given continuous probability distribution $\rho$ we associate its Shannon entropy $S(\rho)=$

$-\int \rho \ln \rho d x$, [25]. If an expectation value $E\left[\ln \left(1+x^{2}\right)\right]$ is prescribed (e.g. fixed), the maximum entropy probability function belongs to a one-parameter family

$$
\rho_{*}(x)=\frac{\Gamma(\alpha)}{\sqrt{\pi} \Gamma(\alpha-1 / 2))} \frac{1}{\left(1+x^{2}\right)^{\alpha}}
$$

where $\alpha>1 / 2,[25]$.

The gamma function $\Gamma(\alpha)=\int_{0}^{\infty} \exp (-t) t^{\alpha-1} d t$ we specialize to integer $\alpha=n+1$ values, with $n \geq 0$. Then $\Gamma(n+1)=n$ ! and $\Gamma(\alpha-1 / 2) \rightarrow \Gamma(n+1 / 2)=[(2 n) ! \sqrt{\pi}] / n ! 2^{2 n}$.

The Cauchy distribution is a special case of the above $\rho_{*}$ that corresponds to $\alpha=1$. The density (40) is the second, $\alpha=2$, member of the $\alpha$-integer hierarchy (we presume $\sigma=1)$.

To elucidate an intimate connection with the underlying Cauchy noise, let us invoke the Fourier transform $\mathcal{F}\left[\rho_{*}\right]$, e.g. a characteristic function of $\rho_{*}$. Namely, we have, [26]:

$$
\mathcal{F}\left[1 /\left(x^{2}+\gamma^{2}\right)^{n}\right]=\frac{(-1)^{n-1} \pi}{(n-1) !} \frac{\partial^{n-1}}{\partial z^{n-1}}\left[\frac{\exp (-\sqrt{z}|p|)}{\sqrt{z}}\right]_{z=\gamma^{2}} .
$$


For the (unnormalized, $\alpha=1$ ) Cauchy density $1 /\left(x^{2}+\gamma^{2}\right)$, we infer $(\pi / \gamma) \exp (-\gamma|p|)$. For the quadratic $(\alpha=2)$ case, we obtain $\left(\pi / 2 \gamma^{2}\right)[|p|+(1 / \gamma)] \exp (-\gamma|p|)$.

Given an invariant probability density of the form (47). Assume that $\mathcal{V}(x)$ can be inferred, e.g. exists, and additionally fits the restrictions of Corollary 2 in Ref. [6].

Then, we can be sure that the corresponding "topological" Cauchy-type process can be consistently defined. For each concrete $\mathcal{V}(x)$, the resulting Markov stochastic process of the jump-type, that is determined by the Cauchy generator plus a suitable potential function, appears to be unique. Specifically, let us consider

$$
\rho_{*}(x)=\frac{16}{5 \pi} \frac{1}{\left(1+x^{2}\right)^{4}} .
$$

By evaluating principal value integrals, we end up with the following expression for the Feynman-Kac (semigroup) potential:

$$
\mathcal{V}(x)=\frac{\gamma}{2\left(1+x^{2}\right)}\left(x^{4}+6 x^{2}-3\right) .
$$

The potential is bounded from below, its minimum at $x=0$ equals $-3 \gamma / 2$. For large values of $|x|$, the potential behaves as $\sim(\gamma / 2) x^{2}$ i.e. shows up a harmonic behavior.

Apart from the unbounded-ness of $\mathcal{V}(x)$ from above, this potential obeys the minimal requirements of Corollary 2 in Ref. [6]: can be made positive (add a suitable constant), is locally bounded (e.g. is bounded on each compact set) and is measurable (e.g. can be arbitrarily well approximated by means of sequences of step functions).

We can readily address the problem (vi) o Section 3.2.3. Be employing the density (49), we get, [26]:

$$
b(x)=-\frac{\gamma x}{16}\left(5 x^{6}+21 x^{4}+35 x^{2}+35\right) .
$$

This a bit discouraging expression shows a linear friction $b \sim-x$ for small $x$ and a strong taming behavior $b \sim-x^{7}$ for large $x$.

\section{Conclusions and prospects}

Insightful, explicitly solvable models are scarce in theoretical studies of Lévy flights, in the presence of external potentials and/or external conservative forces. Therefore, our major task was to find novel examples, that would shed some light on apparent discrepancies between dynamical patterns of behavior associated with two different fractional transport equations (29) and (31) that are met in the literature.

We have demonstrated that so-called topological Lévy processes form a subclass of solutions to the Schrödinger boundary data problem. The pertinent dynamical behavior stems form a suitable Lévy-Schrödinger semigroup. The crucial role of the 
involved Feynman-Kac potential has been identified. We have deduced these potential functions explicitly in a number of cases.

The major gain of those observations is that a mathematical theory of Ref. [6] tells one what are the necessary functional properties of admissible Feynman-Kac potentials. Their proper choice makes a topological Lévy process a well behaved mathematical construct, with a well defined Markovian dynamics and stationary density. That gives clear indications towards any explicit computer-assisted modeling.

Our focus was upon confinement mechanisms that tame Lévy flights to the extent that second moments of their probability densities are admissible. We have proved that the pertinent dynamical patterns of behavior stay in close affinity in the nearequilibrium regime and, in each considered case, admit common for both stationary density. In turn, this density determines a functional form of the above mentioned, dynamical semigroup-defining, potential function.

Our statement of problems involved can be found in Section 3.2.3. We have analyzed problems (i)-(iii) and (vi). A number of important and interesting topics has been left aside in the present paper. Namely, one may choose a priori a simple, physics motivated, functional form of the potential $\mathcal{V}$ (like e.g. $\mathcal{V} \sim x^{2}$ or $\sim x^{4}$ ) and thence the related Lévy and specifically Cauchy semigroup. The major technical problem (to be circumvented by a computer-assisted exploration of the issue) is to deduce an explicit functional form of the corresponding asymptotic invariant density and to visualize dynamical patterns of behavior in the vicinity a stationary state. This topic has not received attention in papers on topological super-diffusions [14]-[17].

Since, the Schrödinger boundary data problem allows for a construction of an interpolating Markovian processes between any two a priori prescribed probability densities, it is of interest to fix an initial density and choose an invariant density as an asymptotic (terminal) datum. A comparison of a detailed temporal behavior of the Langevin-based and affine topological process (both sharing the chosen invariant density) is of interest. This issue is postponed to the future publication.

Acknowledgement: Partial support from the Laboratory for Physical Foundations of Information Processing is gratefully acknowledged. I would like to express my gratitude to Professor Vladimir Stephanovich who detected an error in the previous version of Section 4.2 and cross-checked a number of formulas in the paper.

\section{References}

[1] Lévy Flights and Related Topics in Physics, Lecture Notes in Physics, edited by M. F. Shlesinger, G.M. Zaslavsky, and U. Frisch (Springer-Verlag, Berlin, 1995 
[2] Chaos: The interplay between deterministic and stochastic behavior, edited by P. Garbaczewski, M. Wolf and A. Weron, (Springer-Verlag, Berlin, 1995)

[3] D. Applebaum, Lévy processes and stochastic calculus. Cambridge University Press, 2004

[4] K-I. Sato, Lévy processes and infinitely divisible distributions, Cambridge University Press, 1999

[5] P. Garbaczewski, J. R. Klauder and R. Olkiewicz, Phys. Rev. E 51, 4114, (1995)

[6] P. Garbaczewski and R. Olkiewicz, J. Math. Phys. 40, 1057, (1999)

[7] P. Garbaczewski and R. Olkiewicz, J. Math. Phys. 41, 6843, (2000)

[8] S. Jespersen, R. Metzler and H. C. Fogedby, Phys. Rev. E 59, 2736, (1999)

[9] P. D. Ditlevsen, Phys. Rev. E 60, 172, (1999)

[10] V. V. Janovsky et al., Physica A 282, 13, (2000)

[11] A. Chechkin et al., Chem. Phys. 284, 233, (2002)

[12] A. A. Dubkov and B. Spagnolo, Acta Phys. Pol. A 38, 1745, (2007)

[13] A. A. Dubkov, B. Spagnolo and V. V. Uchaikin, Int. J. Bifurcations and Chaos, 18, 2549, (2008)

[14] V. V. Belik and D. Brockmann, New. J. Phys. 9, 54, (2007)

[15] N. Cufaro Petroni and M. Pusterla, Physica A 388, 824, (2009)

[16] D. Brockmann and I. Sokolov, Chem. Phys. 284, 409, (2002)

[17] D. Brockmann and T. Geisel, Phys. Rev. Lett. 90, 170601, (2003)

[18] D. Brockmann and T. Geisel, Phys. Rev. Lett. 91, 048303, (2003)

[19] J. C. Zambrini, Phys. Rev. A 35, 3631, (1987)

[20] P. Garbaczewski, Acta Phys. Pol. B 40, 1353, (2009)

[21] P. Garbaczewski, Phys. Rev. E 78, 031101, (2008)

[22] M. C. Mackey and M. Tyran-Kamińska, J. Stat. Phys. 124, 1443-1470, (2006)

[23] H. Risken, The Fokker-Planck equation, Springer-Verlag, Berlin, 1989 
[24] I. S. Gradshteyn and I. M. Ryzhik, Table of integrals, series and products, Academic Press, NY, 2007

[25] J. N. Kapur and H. K. Kesavan, Entropy Optimization Principles with Applications, Academic Press, Boston, 1992

[26] V. Stephanovich, private communication 